\title{
Performance Comparison of Various MIMO Detectors in Presence of Gaussian and Non-Gaussian Noise
}

\author{
Jaskirat Kaur \\ Student, M.Tech \\ Dept. of Electronics \& Communication \\ GNDU,RC-Jalandhar,India
}

\author{
Harmandar Kaur \\ Asst. Professor \\ Dept.ofElectronics\&Communication \\ GNDU,RC-Jalandhar,India
}

\begin{abstract}
The Multiple-Input Multiple-Output (MIMO) - Orthogonal Frequency Division Multiplexing (OFDM) technology significantly provides high transmission rate and robustness against multi-path fading and other channel impairments. Mostly, MIMO-OFDM system is analyzed only in presence of additive white Gaussian noise (AWGN) only, but in practice, in many actual wireless environments impulsive characteristics are present. In this paper, the performance of various MIMO detectors is analyzed and compared in presence of both AWGN and impulse noise for different modulations and different antenna configurations of MIMO system.
\end{abstract}

\section{Keywords}

MIMO, OFDM, MIMO Detectors, AWGN

\section{INTRODUCTION}

MIMO technique provides benefits of increased system capacity and spectral efficiency for future wireless communication without increasing the use of spectrum, throughput, reliability, power consumption and less sensitivity to fading. Many authors, in literature, have analyzed MIMO systems in the presence of the ideal Gaussian noise model [1] only, but in practice, in many actual wireless channels, the noise shows impulsive characteristics to some level [2]. The impulsiveness may occur because of many sources such as automobile ignitions, radiation from power lines, electricdevices and multiple access interference.

To mitigate the effect of impulsive noise in MIMO-OFDM system, several methods are provided in literature. It is found that under low SNR assumption, by preceding a conventional OFDM demodulator with memoryless nonlinearity, the adverse effect of impulsive noise gets reduced [3], [4], [5]. In order to mitigate the effect of impulse noise in modern MIMO-OFDM receivers, suboptimal clipping or blanking techniques are used in [6], [7].

In this paper, the performance of various MIMO detectors is analyzed in the presence of both AWGN and impulsive noise. MIMO Linear detectors Zero Forcing (ZF) and Vertical Bell Labs Layered Space-Time (V-BLAST) and non-linear detectors Maximum Likelihood (ML), Sphere Decoder (SD) with 12 norm and SD with $\infty$ - norm are considered for performance analysis.

\section{MIMO DETECTORS}

For MIMO systems, there are numerous detection techniques which include linear detectors such as ZF and V-BLAST and non-linear detectors such as ML detector, SD with norm 2 and SD with norm $\infty$ methods.

\subsection{ZF Detector}

It is one of the simplest detector which acts as an equalizer. It uses an algorithm which apply inverse of the channel frequency response to the received signal, to restore the signal after the channel.

\subsection{V-BLAST Detector}

V-BLAST is a type of linear detector that provides better performance than ZF but with small increase in complexity. The V-BLAST strategy is based on successive interference cancellation. The notion behind this strategy is to use a suitable (space-time) encoding scheme at the transmitter, by using the Ordered Successive Interference cancellation (OSIC) or simply the Successive Interference cancellation (SIC) detector, in order to achieve good performance at the receiver.

\subsection{Detector}

It is an optimum non-linear detector. It searches throughout the lattice points, compares the received signals with all possible transmitted signal vectors $\mathrm{s}$ and determines the transmit symbol vector $\widehat{s}$ according to the ML Principle.

\subsection{Sphere Decoder}

The SD algorithm searches, within the sphere of chosen radius, for the closest lattice point to the received signal, where each lattice point within a lattice field represents a codeword.

SD uses 12 norm to conduct the algorithm. In [8], it is observed that $\infty$ - norm can be used for SD, which reduces the hardware complexity of SD but incurs small amount of loss in performance [9].

\section{IMPULSIVE NOISE IN MIMO-OFDM SYSTEM}

Impulse noise 2is an additive interference which is active only for short durations. Because of small duty cycle of impulse noise, the average power of impulse noise is much lower than its instantaneous power, because of its small duty cycle during active intervals. This results in a large peak-to average ratio (PAR) - the key characteristic of impulse noise [10]. Even in case of high SNRs, the impulse noise can severely deteriorate the performance and the reliability of wireless 
communications systems. In order to defend against undesirable performance, the true characteristics of the noise must be considered.

Impulsive, non-Gaussian noise is accepted in many communication environments such as radio frequency interference (RFI) generated by computers for embedded wireless data transceivers, RFI in indoor and outdoor channels and co-channel interference in a Poisson field of interferers due to a variety of sources, such as atmospheric noise, manmade electromagnetic interference, power lines, closely located wireless systems or ignition noise [11].

Also in indoor wireless environment, devices with electromechanical switches such as printers, photocopy machines, electrical motors in elevators and refrigerator units are considered as impulse noise sources. Moreover, microwave ovens, drilling machines, cash register receipt printers, car ignitions, gas-powered engines, trains and welding etc. cause impulse noise in frequency bands which concur with the currently utilized frequencies of modern wireless networks [12].

\subsection{Sources of Impulse Noise}

There are mainly three potential sources of impulsive RFI in the wireless networks, which are:

\subsubsection{Natural Source}

This type of noise occurs mainly because of natural phenomena such as lightning and radiation from the sun, extraterrestrial solar and cosmic sources etc. Usually, this type of noise is called atmospheric noise.

\subsubsection{Inherent Source}

Inherent noise is the noise within electronic equipment.

\subsubsection{Man-made source}

Many sources of impulse noise are produced by a variety of electrical and electronic equipment and systems. These sources of impulse noise include high power broadcast systems. Man-made sources are further of two types: indoor source and outdoor sources.

\subsubsection{Indoor sources}

These sources include domestic appliances which produce frequent impulse noise. For example, house appliances such as irons, dish washers, kettles, refrigerator units, ovens, photocopy machines, elevator, food mixers, electric razors, drills, washing machines, central heating thermostats and light switches are common sources of impulse noise [13].

\subsubsection{Outdoor sources}

In outdoor environments, gas-powered engines, car ignition, welding, compressor motors, high power grid lines, corona effects and medical equipment are remarkable sources that produce impulsive noise [12].

\section{SIMULATION RESULTS AND DISCUSSION}

The performance of MIMO detectors ZF, V-BLAST, ML, SD with 12 norm, SD with $l^{\infty}$ norm is evaluated in presence of AWGN and impulse noise for modulation M-QAM $(\mathrm{M}=4,8,16)$ and for different MIMO antenna configurations. All simulations are performed in MATLAB 2014a software.

\subsection{Performance Evaluation of $\mathrm{ZF} \& \mathrm{~V}$ - BLAST in Presence of AWGN and Impulse Noise for Modulation M-QAM $(M=4,8,16)$}

Here two cases are considered for ZF and V-BLAST: one is the system in presence of AWGN only and other is system in presence of both AWGN and impulsive noise.

For $16 \times 16$ MIMO, performance evaluation of $\mathrm{ZF}$ in presence of AWGN and Impulse noise for modulation M-QAM, $(\mathrm{M}=$ $4,8,16$ ) is shown in fig. 1 .

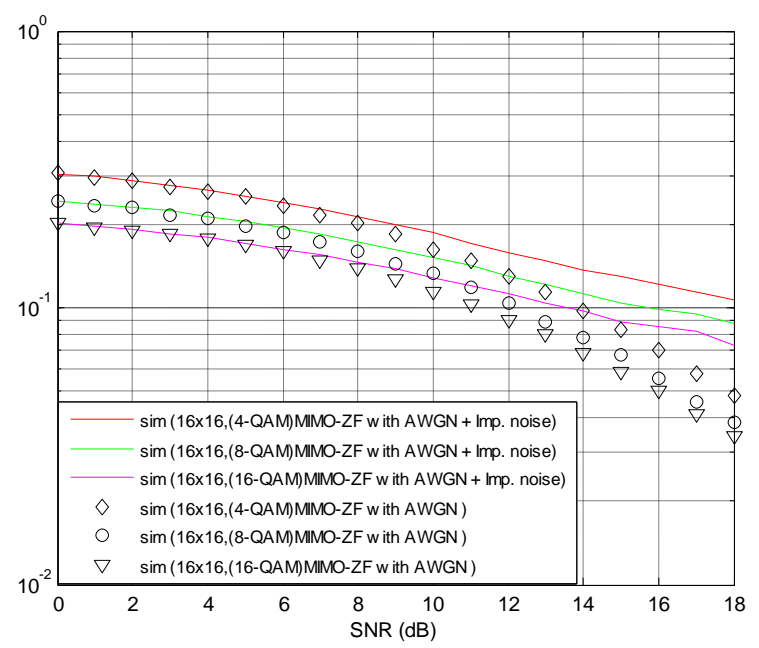

Fig.1 Performance evaluation of 16×16 MIMO ZF system for different $M$ in presence of AWGN \& impulse noise

The SNR values for MIMO-ZF system at BER $=0.1$ are given in table 1 .

Table 1: Table showing SNR values for $16 \times 16$ MIMO-ZF with different modulations at $B E R=0.1$

\begin{tabular}{|c|c|c|c|}
\hline \multirow{2}{*}{ Type of Noise Present } & \multicolumn{3}{|c|}{ SNR(dB) } \\
\cline { 2 - 4 } & 4-QAM & $\begin{array}{c}8- \\
\text { QAM }\end{array}$ & $\begin{array}{c}16- \\
\text { QAM }\end{array}$ \\
\hline AWGN & 14 & 12 & 11 \\
\hline $\begin{array}{c}\text { AWGN + Impulse } \\
\text { Noise }\end{array}$ & $\begin{array}{c}\text { More than } \\
18\end{array}$ & 16 & 14 \\
& & & \\
\hline
\end{tabular}

From results in table 1, it is shown that the performance of MIMO-ZF system in presence of AWGN and impulse noise is poor as compared to MIMO-ZF system with only AWGN. In both cases, MIMO-ZF with 16-QAM provides better performance results as compared to both 8-QAM and 4-QAM modulations.

For $16 \times 16$ MIMO, performance evaluation of V-BLAST in presence of AWGN and impulse noise for modulation $\mathrm{M}$ QAM, $(\mathrm{M}=4,8,16)$ is shown in fig.2. 


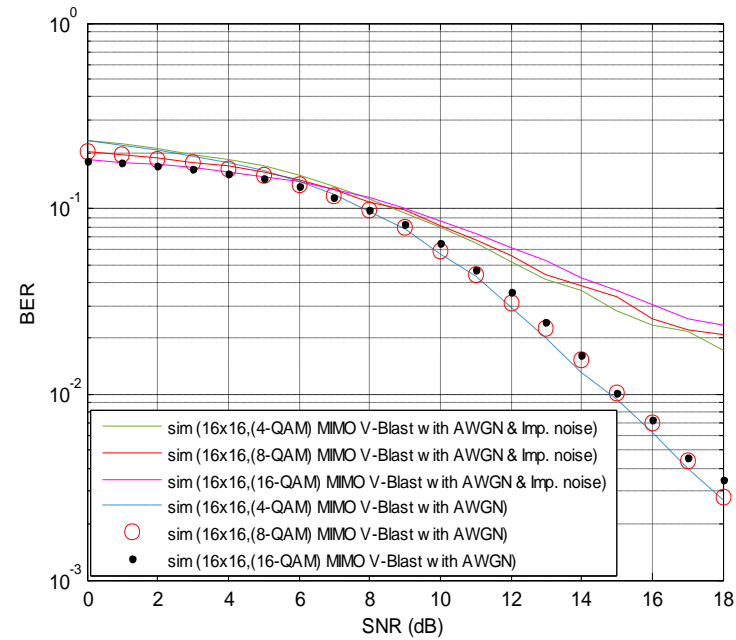

Fig.2 Performance evaluation of $16 \times 16$ MIMO V-BLAST system for different $M$ in presence of AWGN and impulse noise

It is clear from fig. 2 that for SNR $=0-8 \mathrm{~dB}$, the performance of MIMO-V-BLAST system in presence of AWGN and impulse noise is poor as compared to system with only AWGN. For SNR > 8dB, in both cases, 4-QAM modulation provide good system performance as compared to other modulations.

\subsection{Performance Comparison of $\mathrm{ZF}$ and $\mathrm{V}$ - BLAST for Different Modulations in Presence of AWGN And Impulse Noise}

The performance of ZF and V-BLAST is compared in terms of BER and SNR in fig.4.3. The results are obtained using different M-QAM modulations for $16 \times 16$ antenna configuration of MIMO system.

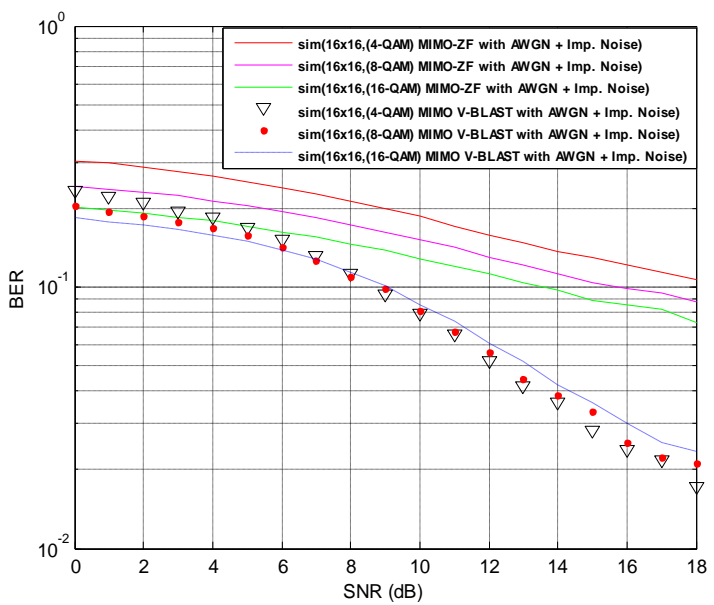

Fig.3 Performance comparison of ZF and V-BLAST for $16 \times 16$ MIMO system using different M-QAM in presence of AWGN and impulse Noise

The results in fig. 3 show that V-BLAST shows good BER performance as compared to ZF for all M-QAM modulations. In case of ZF, 16 QAM modulation provides good system performance as compared to other modulations. In case of $\mathrm{V}$ BLAST, for SNR < 6dB, 16 QAM modulation provides good system performance as compared to other modulations; for $\mathrm{SNR}=6-9 \mathrm{~dB}$, all modulations provide same system performance; for SNR > 9dB, 4 QAM modulation provides good system performance as compared to other modulations.

\subsection{Performance Comparison of $\mathrm{ZF}$ and $\mathrm{V}$ - BLAST for Different Antenna Configurations $(4 \times 4,8 \times 8,16 \times 16)$ of MIMO System in Presence of AWGN and Impulse Noise}

The performance of ZF and V-BLAST is compared in terms of BER and SNR in fig.4. The results are obtained for different antenna configurations of MIMO system using 4QAM modulation.

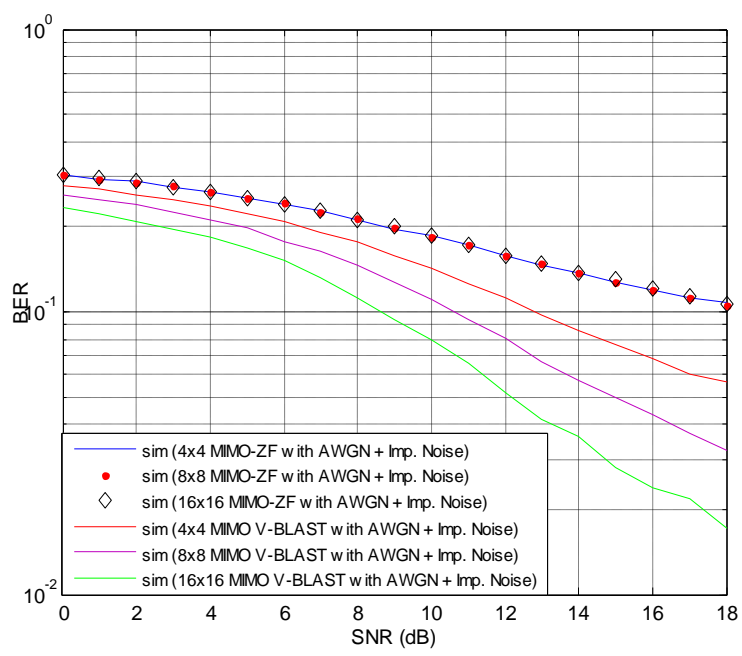

Fig.4 Performance comparison of ZF and V-BLAST using different antenna configurations for 4-QAM modulation in presence of AWGN \& impulse Noise

The SNR values for V-BLAST and ZF at BER $=0.1$ are given in table 2 .

Table 2: Table showing SNR values for V-BLAST and ZF with different modulation schemes for $16 \times 16$ MIMO system

\begin{tabular}{|c|c|c|}
\hline \multirow{2}{*}{ Antenna Configuration } & \multicolumn{2}{|c|}{ SNR(dB) } \\
\cline { 2 - 3 } & V-BLAST & ZF \\
\hline $4 \times 4$ & 13 & approx.18dB \\
\hline $8 \times 8$ & & \\
\hline $16 \times 16$ & 10.8 & approx.18dB \\
\hline & 8.6 & approx.18dB \\
\hline
\end{tabular}

From results in table 2, it is shown that V-BLAST shows good BER performance as compared to $\mathrm{ZF}$ for all antenna configurations of MIMO system. As size of antenna configuration increases, V-BLAST shows more good performance and ZF shows same performance for all antenna configurations of MIMO system using same modulation 4QAM. 


\subsection{Performance Comparison of ML, SD with Norm 2, SD with $\infty$ - Norm, ZF and V- BLAST for Different Modulations in Presence of AWGN \& Impulse Noise}

The performance of ML, SD with norm 2, SD with norm $\infty$, $\mathrm{ZF}$ and V-BLAST is compared in terms of BER and SNR in fig.5. The results are obtained for $4 \times 4$ antenna configuration using 4-QAM modulation.

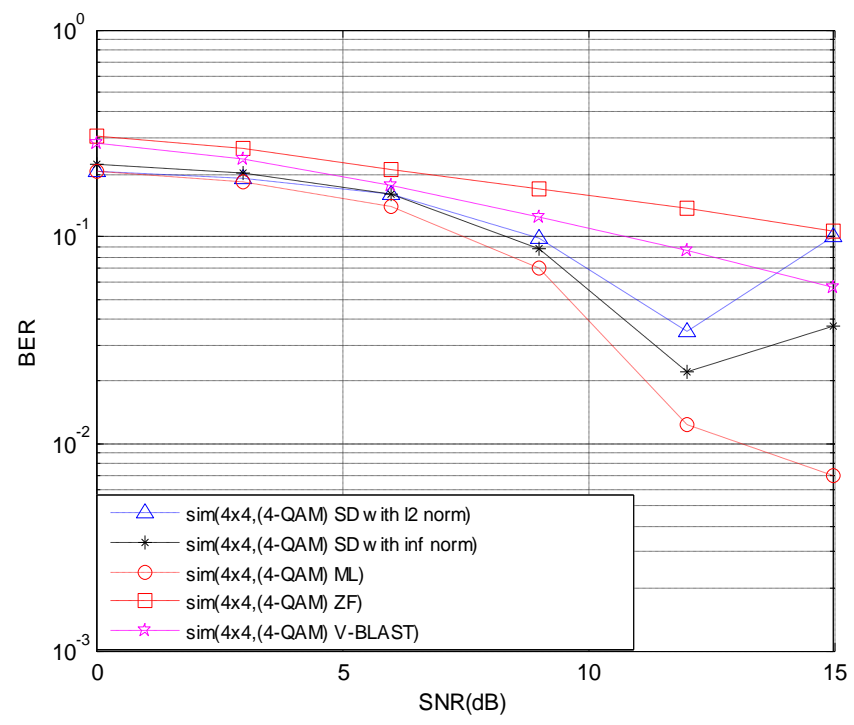

Fig.5 Performance comparison of SD with norm 2, SD with $\infty$ - norm, ML, ZF and V-BLAST for $4 \times 4$ MIMO system using 4-QAM modulation in presence of AWGN and impulse Noise

The SNR values for ML, SD with norm 2, SD with $\infty-$ norm, $\mathrm{ZF}$ and V-BLAST at BER $=0.1$ are given in table 3.

Table 3: Table showing SNR values for various MIMO detectors at $B E R=0.1$ for $(4-Q A M), 16 \times 16$ MIMO system

\begin{tabular}{|c|c|}
\hline Type of detector & SNR(dB) \\
\hline SD with norm 2 & 9 \\
\hline SD with $\infty$ - Norm & 8 \\
\hline ML detector & 7.5 \\
\hline ZF detector & 15 \\
\hline V-BLAST detector & 11 \\
\hline
\end{tabular}

From results in table 3, it is shown that ML detector shows the best performance as compared to all other detectors. The order of performance for MIMO detectors in decreasing order is $\mathrm{ML}>\mathrm{SD}$ with norm $\infty>\mathrm{SD}$ with norm $2>\mathrm{V}$-BLAST $>\mathrm{ZF}$.

The performance of ML, SD with norm 2, SD with norm $\infty$, $\mathrm{ZF}$ and V-BLAST is compared in terms of BER and SNR in fig.6. The results are obtained for $4 \times 4$ antenna configuration using 16-QAM modulation.

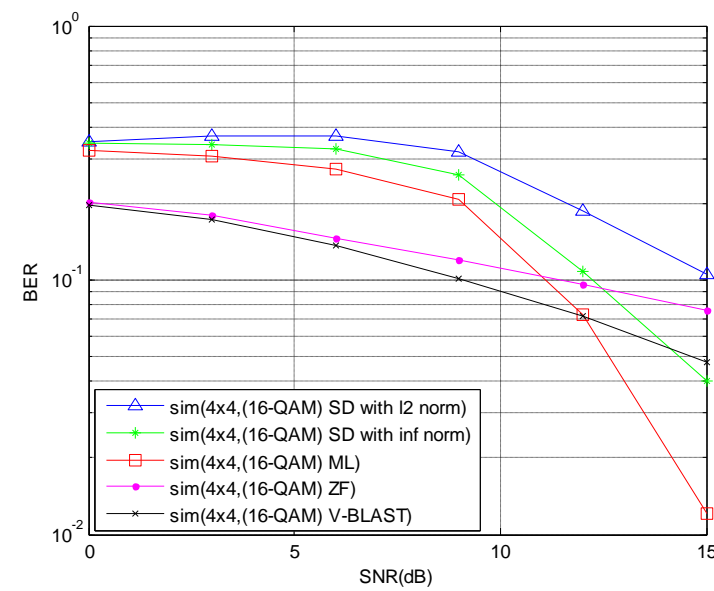

Fig.6 Performance comparison of SD with norm 2, SD with $\infty$ - norm, ML, ZF and V-BLAST for $4 \times 4$ MIMO system using 16-QAM modulation

It is clear from fig.6, for $S N R=0-11 d B$, the order of performance for MIMO detectors in decreasing order is $\mathrm{V}$ BLAST $>$ ZF $>$ ML > SD with norm $\infty>\mathrm{SD}$ with norm 2; for $S N R=11-12 d B$, the order of performance for MIMO detectors in decreasing order is V-BLAST $>\mathrm{ML}>\mathrm{ZF}>\mathrm{SD}$ with norm $\infty>\mathrm{SD}$ with norm 2; for $S N R \geq 12-14 d B$, the order of performance for MIMO detectors in decreasing order is $\mathrm{ML}>\mathrm{V}$-BLAST $>\mathrm{SD}$ with norm $\infty>\mathrm{ZF}>\mathrm{SD}$ with norm 2 ; for $S N R>14 d B$, the order of performance for MIMO detectors in decreasing order is $\mathrm{ML}>\mathrm{SD}$ with norm $\infty>\mathrm{V}$ BLAST $>$ ZF $>$ SD with norm 2 .

\section{CONCLUSION}

In this paper, the impact of impulse noise in MIMO-OFDM system is described. The performance of various MIMO detectors is analyzed and compared for different modulations and different antenna configurations of MIMO system in presence of both AWGN and impulse noise. From simulation results conducted in presence of both AWGN and impulse noise, it is concluded that in case of linear detectors, V-Blast shows better performance than ZF for all modulations and antenna configurations. In case of non-linear detectors, ML shows better performance than SD for all modulations using $4 \times 4$ MIMO system. On comparing performance of all detectors, it is concluded that for 4-QAM, 4×4 MIMO system, ML shows the best performance and for 16-QAM, $4 \times 4$ MIMO system, V-BLAST and ML shows the best performance at low and high SNR values respectively.

\section{REFERENCES}

[1] S. M. Alamouti, "A Simple Transmit Diversity Technique for Wireless Communications," IEEE J. Sel. Areas in Comms., vol.16, no.8, pp. 1451- 1458, Oct. 1998.

[2] A. Li et al., "Receiver Design of MIMO Systems in a Mixture of Gaussian Noise and Impulsive Noise," Vehicular Technology Conference (VTC), 2004. IEEE International Conference on On page(s): 1493 - 1497, Vol.2, 2004.

[3] O. P. Haffenden et al., "Detection and removal of clipping in multicarrier receivers," European patent application EP1043874, Oct. 11, 2000, published in Bulletin 2000/41. 
[4] O. Antonov, "Optimal detection of signals in nonGaussian noise," RadioEng. Electron. Phys. (USSR), vol. 12, pp. 541-548, 1967.

[5] S. S. Rappaport and L. Kurz, "An optimal nonlinear detector for digital data transmission through nonGaussian channels," IEEE Trans. Commun. Technol., vol. COM-14, pp. 266-274, June 1966.

[6] N. P. Cowley, A. Payne and M. Dawkins, "COFDM tuner with impulse noise reduction," European patent application EP1180851, Feb. 20, 2002, published in Bulletin 2002/08.

[7] H. A. Suraweera, C. Chai, J. Shentu and J. Armstrong, "Analysis of impulse noise mitigation techniques for digital television systems," in Proc. 8th International OFDM Workshop, Sept. 2003, pp. 172-176.

[8] A. Burg, M. Borgmann, M. Wenk, M. Zellweger, W. Fichtner, and H. B"olcskei, "VLSI implementation of MIMO detection using the sphere decoding algorithm," IEEE J. of Solid-State Circuits, vol. 40, no. 7, pp. 15661577, July 2005.
[9] D. Seethaler and H. Bolcskei, "Infinity-Norm SphereDecoding," IEEE ISIT, presented in part at IEEE ISIT, Toronto, in Canada, July 2008.

[10] D. Middleton, "Non-Gaussian Noise Models in Signal Processing for Telecommunications: New Methods and Results for Class A and Class B Noise Models," IEEE Trans. On Info. Theory, vol. 45, no. 4, pp. 1129-1149, 1999.

[11] K. A. Saaifan and W. Henkel, "A Receiver Design for MIMO Systems over Rayleigh Fading Channels with Correlated Impulse Noise," IEEE Trans., Globecom 2012, pp. 2481-2486, 2012.

[12] M. I. Hossain, "Effect of Impulse Noise in Wireless Communications," a report, Comm. Systems and Elec., Jacobs University, Bremen, 2012.

[13] K.L. Blackard et al., "Measurements and Models of Radio Frequency Impulsive Noise for Indoor Wireless Communications," IEEE Journal on Selected Areas in Comms., Vol. 11, No. 7, September 1993. 